an organic matrix dominated by collagen, with nanoscale inorganic crystals composed mostly of hydroxyapatite. These two phases are arranged in a staggered nanostructure of largeaspect-ratio inorganic crystals embedded in the organic matrix. In the June 14 issue of Chemistry of Materials (p. 3059; DOI: $10.1021 / \mathrm{cm} 050492 \mathrm{k}), \mathrm{C}$. Jaeger of the Federal Institute of Materials Research and Testing in Berlin, Germany; N.S. Groom of the University of Cambridge; E.A. Bowe of the Royal Veterinary College in London; R.C. Murray of the Animal Health Trust in Suffolk; and their colleagues have reported solid-state nuclear magnetic resonance (NMR) studies of the interface of the organic phase and inorganic crystallites in bone.

The researchers believe that understanding these organic-inorganic interface properties is the key to understanding the macroscopic material properties of bone.

"Not only would such knowledge help us understand the superior properties of bone, but it may also assist in the design of new, synthetic bone-like and other materials," said coresearcher M.J. Duer of the Department of Chemistry at the University of Cambridge.

Based on a series of NMR experiments, the scientists proposed a quantitative structural model of the possible binding site for a carboxylate group at the surface of the calcium phosphate phase in bone. The results demonstrated that the glutamate binding proteins interact very strongly with $\mathrm{Ca}^{2+}$ ions on the surface of the mineral phase.

"As collagen itself contains very little glutamate or glutamine, the proteins associated with binding to the mineral phase must be in the non-collageneous part of the protein matrix," said Duer. These results suggest that the collagen itself does not bind to the mineral particles, but that an additional protein interface layer is responsible for the binding.

"Since the $\mathrm{P}-\mathrm{OH}$ groups of the inorganic platelets are sufficiently close to the carboxylate or carbonyl groups of the organic material, hydrogen bonds may play a critical role in determining the interface properties," said Duer. The researchers report that this hypothesis is further supported by rotational echo double resonance (REDOR) experiments.

MARKUS J. BUEHLER

\section{"Top-Down" Technique Allows for PRINTing of Shape-Specific Nanobiomaterials}

In recent years, biomaterials have played a pivotal role in the success of drug delivery and medical devices. To achieve optimal performance in drug, targeting, and recognition applications, these materials should exhibit programmed responses to external stimuli and recognition events. Examples of such materials are socalled "smart" particles, whose form (i.e., morphology and composition) dictates function. In the June 2 issue of the Journal of the American Chemical Society (DOI: 10.1021/ja05/977c), J.P. Rolland, B.W. Maynor, L.E. Euliss, A.E. Exner, G.M. Denison, and J.M. DeSimone of the University of North Carolina at Chapel Hill outline a method for fabricating arrays of these particles using a "top-down" approach called PRINT (particle replication in nonwetting templates). Unlike traditional imprint lithography techniques, PRINT uses a nonwetting layer of fluorinated surfaces to confine a liquid precursor inside the features of a photocurable perfluoropolyether (PFPE) mold, allowing for the production of isolated particles. The researchers said that the technique enables the fabrication of repeatable features at dimensions of $100 \mathrm{~nm}$ with control over particle size, shape, and composition.

The researchers started with master templates that were either obtained commercially or fabricated in silicon using electronbeam lithography. PFPE replica molds were then made by photochemically curing a dimethacrylate-functionalized PFPE oligomer

\section{See More... \\ Do More...}

\section{Integrated Solutions for Integrated Applications}

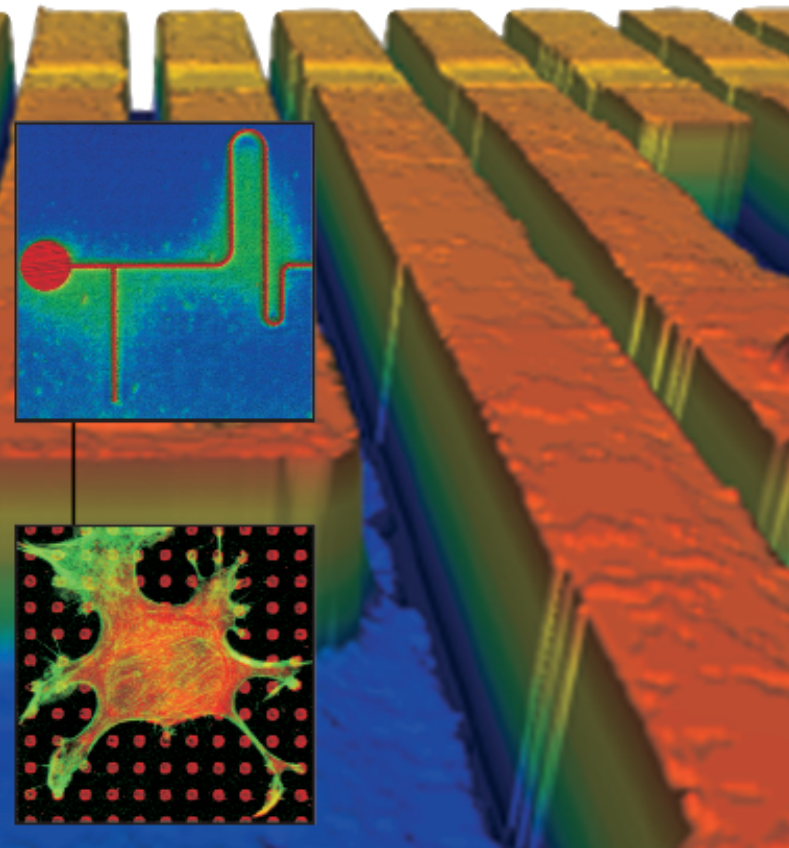

\section{Carl Zeiss LSM 5 PASCAL}

Confocal Laser Scanning Microscope

Insight into Biology and Materials Science through:

- 3D Imaging

- Surface topography

- Volumetric measurement

- Multi-label Imaging

- Fluorescence \& Reflected light imaging

Applications in Research and Quality Assurance:

- Microfluidics

- Microarrays

- Microreplication

- Sensors

- Lab-on-Chip

Carl Zeiss Microlmaging Inc.

Thornwood, NY 10594

800.233 .2343

micro@zeiss.com

zeiss.com/lsm-mat

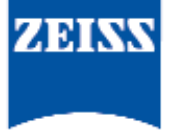

We make it visible. 

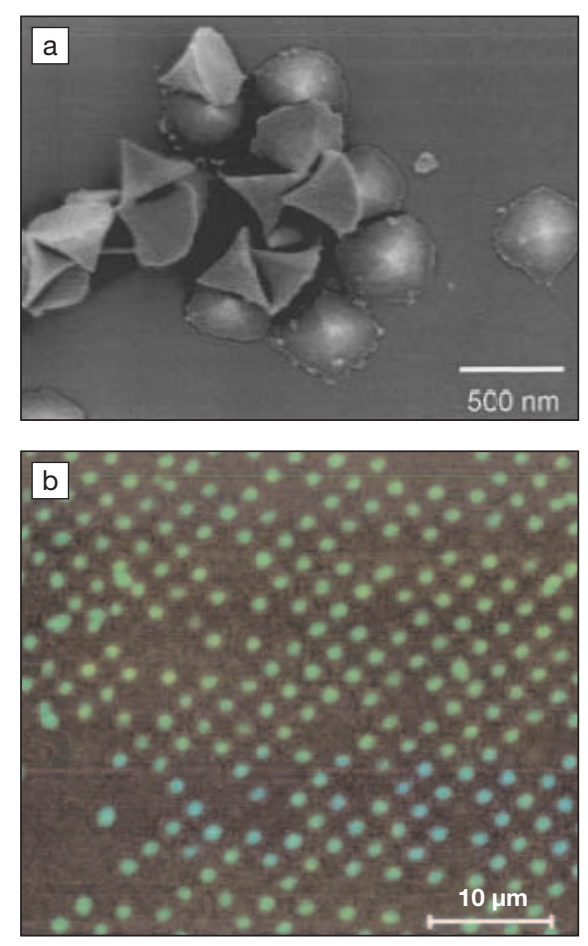

Figure 1: Conical-shaped particles made using the PRINT (particle replication in nonwetting templates) process: (a) scanning electron micrograph of conical triacrylate particles, $<50 \mathrm{~nm}$ at the tip, mechanically harvested from an array by using a doctor blade;

(b) fluorescent confocal micrograph of $500 \mathrm{~nm}$ conical-shaped poly(ethylene glycol) acrylate particles containing avidin, tagged with fluorescent dye.

that was pooled onto one of the masters. The researchers demonstrated the fabrication of monodisperse particles of technologically relevant materials such as poly(ethylene glycol acrylate), or PEGacrylate; bioabsorbable poly(lactide); and triacrylate resin. PRINT allows a variety of particles to be created, ranging from triacrylate nanoparticles ( $200 \mathrm{~nm}$ in size) to complex micrometer-scale objects such as fluorescently labeled avidin (a protein) encapsulated in conical PEG-acrylate particles, shown in Figure 1. Vacancies are sometimes observed in regular arrays because material that was meant to be deposited remains trapped in the walls of the mold, but the researchers are working on methods to ensure release of the material to make error-free arrays. The researchers said that the PFPE-elastomeric molds are robust, having been used for multiple experiments with no deterioration. The ability to encapsulate delicate cargos, such as DNA, proteins (e.g., avidin), and chemotherapeutics (e.g., doxorubicin) within particles will make PRINT an important technique for next-generation particulate drug-delivery agents, the researchers said.

ADITI RISBUD

\section{UV Irradiation Combined with $\mathrm{TiO}_{2}$ Photocatalyst Kills Bacteria in Flowing Air Streams}

Recent outbreaks of diseases such as SARS and pneumonia, caused by airborne pathogens, has led to great interest in methods for decontaminating bacteriacontaining air, especially in closed environments such as hospitals, factories, public buildings, and aircraft. $\mathrm{TiO}_{2}$ shows strong oxidizing ability under UV irradiation and is commonly used to decompose organic pollutants in air and water. However, only recently has the pathogenkilling power of UV-irradiated $\mathrm{TiO}_{2}$ been explored, with bactericidal effects demonstrated in aqueous suspensions of $\mathrm{TiO}_{2}$ or in cell suspensions deposited on substrates coated with $\mathrm{TiO}_{2}$ thin films. In the June 13 issue of Chemical Communications (p. 2918; DOI: 10.1039/b503638k), V. Keller and colleagues from Louis Pasteur University (Strasbourg, France) report a method to harness this photocatalytic ability of $\mathrm{TiO}_{2}$ to decontaminate a bacteriacontaining air stream.

The researchers used a specially designed air-decontamination device, consisting of an aerosol generator and a bacteria cultivation medium, to provide a steady stream of bacteria-containing air, and a high-surface area UV photoreactor with a specific geometry. A $5 \mu \mathrm{m}$ uniform coating of the titania photocatalyst (80:20 mixture of anatase and rutile phases) was prepared on the inner surface of a Pyrex tube, which had a geometry designed to maximize contact (surface area, $\sim 50 \mathrm{~m}^{2} / \mathrm{g}$ ) between the pathogen-containing air stream and the photocatalyst. Keller and co-workers used Escherichia coli as the model bacteria, because of their similar size and morphology to Legionella pneumophila, and both are Gram-negative bacteria. The researchers carried out photocatalysis using $380 \mathrm{~nm}$ light from black-light tubes surrounding the reactor. The number of live bacteria was determined by collecting samples before and after the photocatalytic reactor. The stain 5-cyano-2,3ditolyl tetrazolium chloride was used as a fluorescent indicator to identify breathing and dead bacteria through fluorescence microscopy. Furthermore, the researchers said that both UV irradiation and $\mathrm{TiO}_{2}$ were essential for the removal of the bacteria; the process did not work with only UV irradiation or only $\mathrm{TiO}_{2}$.

The photocatalytic reactor showed a
99.1-99.8\% efficiency in removing bacteria from flowing air streams, even at high flow rates (1.5-6 $\mathrm{L} \mathrm{min}^{-1}$ ) with up to 26,000 colony-forming units (CFUs) of bacteria per cubic meter, demonstrating that this device could potentially be used for on-line applications in contaminated areas. The researchers said that this technique represents a rapid, easy, and practical route toward addressing risks to public health from microbial pathogens.

SARBAJIT BANERJEE

\section{All-Solid-State Laser Shines in the Orange, Yellow, and Green}

Although diode lasers offer some important capabilities, there is still no widely available, all-solid-state laser system that can be easily tuned over a large range of visible wavelengths. However, thanks to a team of researchers from Macquarie University in Australia, that may soon change. The researchers, R.P. Mildren, H.M. Pask, H. Ogilvy, and J.A. Piper, have demonstrated an all-solid-state laser that can be easily tuned to discrete orange, yellow, and green wavelengths while achieving powers of more than a watt.

As reported in the June 15 issue of Optics Letters (p. 1500), the researchers based their laser system on existing techniques for yellow-orange lasers currently used in medical and defense applications. They began by pumping a neodymium-doped yttrium aluminum garnet (Nd:YAG) crystal with $808 \mathrm{~nm}$ light, generating nearinfrared light at $1064 \mathrm{~nm}$. The crystal was placed in an optical cavity with a slab of $\mathrm{KGd}\left(\mathrm{WO}_{4}\right)_{2}$, which Raman-shifted some of the Nd:YAG light to $1159 \mathrm{~nm}$ and $1272 \mathrm{~nm}$. The novel aspect of the system is the presence of two nonlinear lithium triborate (LBO) crystals in the cavity, to perform frequency mixing of the fundamental and Raman-shifted wavelengths. Frequency mixing, which is used to combine lowenergy, long-wavelength light fields to produce higher-energy, shorter-wavelength light, is usually performed with a single crystal, and strongly depends on the crystal's temperature and angular placement, making it difficult to tune. With the innovative use of two independently temperature-controlled LBO crystals placed in the cavity at different angles, the research team was able to use frequency mixing to selectably convert the near-infrared light to $532 \mathrm{~nm}$ (green), $555 \mathrm{~nm}$ (greenish-yellow), $579 \mathrm{~nm}$ (yellow), and $606 \mathrm{~nm}$ (orange) with high efficiency. The scheme required that the crystal temperatures be tuned over a relatively small range $\left(19^{\circ}-95^{\circ} \mathrm{C}\right)$, and generated $0.25-1.5 \mathrm{~W}$ of optical power. This laser system is very promising for potential commercial applications because of the 